\title{
The acquisition of category structure in unsupervised learning
}

\author{
AUDREY S. KAPLAN and GREGORY L. MURPHY \\ University of Illinois, Urbana, Ilinois
}

\begin{abstract}
Four experiments examined the extent to which prior knowledge influences the acquisition of category structure in unsupervised learning conditions. Prior knowledge is general knowledge about a broad domain that explains why an object has the features it does. Category structure refers to the statistical regularities of features within and across categories. Subjects viewed items and then divided them up into the categories that seemed most natural. Each item had one feature that was related to prior knowledge and five features that were not. The results showed that even this small amount of prior knowledge helped subjects to discover the category structure. In addition, prior knowledge enhanced the learning of many of the category's features, and not just the features that were directly relevant to the knowledge. The results suggest that prior knowledge may help to integrate the features of a category, thereby improving the acquisition of category structure.
\end{abstract}

When people learn a new category, they are influenced by their prior knowledge of the domain that the new category is in. People learn new categories all the time: Novel artifacts are constantly being invented, scientific discoveries carve out previously unknown classes in our world, and education introduces us to new kinds of things. One mechanism by which people acquire these new categories is by relating their prior knowledge of similar objects to the novel categories. For example, when people first learned about space shuttles, they probably used their knowledge of airplanes, rockets, and satellites to understand what space shuttles were. In this article, we are concerned with establishing in greater detail how prior knowledge is used when acquiring new categories. By prior knowledge we refer to knowledge about a broad domain, such as mammals, birds, vehicles, or computers, that exists before learning a new category. This knowledge connects features of a category, perhaps through causal relations, and provides some sort of explanation of why the category has the properties it does (see Carey, 1985; Keil, 1989; Murphy, 1993; Murphy \& Medin, 1985). To give a very simple example (see Kaplan \& Murphy, 1998), people have some idea about how animals fly that provides a possible explanation for why something that has wings can live high on a cliff-the wings explain flying, which in turn explains why this habitat is possible.

What makes something a category? The classical view of categories posited that categories could be defined by singly necessary and jointly sufficient features (see Smith

This research was supported by a National Science Foundation graduate fellowship to the first author and by NIMH Grant MH41704. The authors are grateful to Brian Ross and the reviewers for helpful comments. Correspondence should be addressed to G. L. Murphy, Beckman Institute, University of Illinois, 405 N. Mathews Ave., Urbana, IL 61801 (e-mail: glmurphy@uiuc.edu).
\& Medin, 1981). While definitions may work for some strictly defined categories, such as geometric shapes (e.g., squares and circles can be rigidly defined in terms of lines and angles), most categories do not have these kinds of criterial features. More recently, family resemblance (FR) views (Rosch \& Mervis, 1975) have proposed that category members tend to have many features in common. Thus, it is acknowledged that no unique combination of features is sufficient to describe a category. Instead, different individuals in a category are thought to have some subset of their features in common with members of the same category and some features in common with members of contrasting categories. For example, most birds sing, fly, and have wings, but there are birds that lack one or more of these features (e.g., penguins do not fly), and some nonbirds contain some of these features (e.g., bats fly). The shared features of FR category members support the powerful inductive utility of categories. Knowing that a new object is a member of some category licenses inferences about its nonobvious properties. This is possible because if an object has known features that allow it to be classified correctly, people can use their knowledge of the category to infer that it has other features that are typically correlated with the known features. For example, if someone heard about a new winged animal discovered in the jungle, they might infer that it is a bird and, therefore, that it flies and lays eggs. If bird were represented solely by a few defining features like "has feathers and a beak," such inferences would not be possible.

In the present experiments, we asked how it is that people learn the FR structure of categories without supervision - that is, without feedback about what category each item is in. It is not uncommon for people to identify a new category on their own, such as when one notices a new kind of bird or music or flower that one has not noticed before. In such cases, people identify different ob- 
jects as being the same kind of thing, though not a kind that they knew of before. Later, they may ask or be told what the name is for this new kind of thing. In our experiments, subjects similarly observed items without any external information and then were asked to form categories of the items or to make inferences about an item's features. In the experiments, we explored how prior knowledge influences people's ability to extract the category structure and investigated more precisely what the relationship is between prior knowledge and what subjects learn about the categories.

\section{Prior Knowledge and Category Acquisition}

During category learning, prior knowledge interacts with category structure: the empirical or statistical information about a category of the sort "most of the category members have wings" or "one of the exemplars has a red back, wings, and three legs." When the structure of a category is consistent with the prior knowledge structure, the category is easier to learn than when the category structure and the prior knowledge are inconsistent $\mathrm{Ka}$ plan \& Murphy, 1998; Pazzani, 1991; Wattenmaker, Dewey, T. D. Murphy, \& Medin, 1986). Category and knowledge structures are consistent when the category's content matches people's expectations about how the features and exemplars are related. They are inconsistent when the categories contain contradictions of prior knowledge (e.g., an animal without wings that flies).

Most of the studies concerning prior knowledge effects in categorization have employed supervised learning tasks in which the learner is shown labeled examples of what is and is not in the category (Murphy \& Allopenna, 1994; Pazzani, 1991; Wattenmaker et al., 1986; Wisniewski, 1995). However, people sometimes learn new categories by observing the world and noticing the different kinds of things in it without explicit instruction. Category construction experiments investigate this second type of category learning. In a category construction experiment, subjects are presented with a group of exemplars written on cards, and they are asked to sort the cards into two groups in whatever way seems best or most natural. Table 1 shows the abstract structure for a pair of FR categories used in such a study (Medin, Wattenmaker, \& Hampson, 1987). Each column, labeled D1-D4, refers to a dimension that can be instantiated as one of two features, denoted here by $0 \mathrm{~s}$ and $1 \mathrm{~s}$. Exemplars, or category members, are defined by their values

Table 1

Sample Category Structures Used by Medin, Wattenmaker, and Hampson (1987)

\begin{tabular}{|c|c|c|c|c|c|c|c|c|c|}
\hline \multicolumn{5}{|c|}{ Category A } & \multicolumn{5}{|c|}{ Category $\mathrm{B}$} \\
\hline Exemplar & D1 & D2 & D3 & D4 & Exemplar & $\mathrm{D} 1$ & D2 & D3 & D4 \\
\hline 1 & 1 & 1 & 1 & 1 & 6 & 0 & 0 & 0 & $\overline{0}$ \\
\hline 2 & 0 & 1 & 1 & 1 & 7 & 1 & 0 & 0 & 0 \\
\hline 3 & 1 & 0 & 1 & 1 & 8 & 0 & 1 & 0 & 0 \\
\hline 4 & 1 & 1 & 0 & 1 & 9 & 0 & 0 & 1 & 0 \\
\hline 5 & 1 & 1 & 1 & 0 & 10 & 0 & 0 & 0 & 1 \\
\hline
\end{tabular}

on these four dimensions. Thus, Exemplars $1-5$ comprise one category, and Exemplars 6-10 constitute the contrasting category. The $1 \mathrm{~s}$ and $0 \mathrm{~s}$ are called characteristic features because, for a given category and dimension, the feature represented by either 0 or 1 is more characteristic of the category. However, these features are not defining, because they appear in both categories, albeit with greater frequency in one category than the other. Thus, knowing that an item has the feature " 1 " on the first dimension means it is most likely a member of Category A (Exemplar 1, 3, 4, or 5), but it could also be a member of Category B (Exemplar 7). Because these are FR categories, exemplars in the same category tend to share features (e.g., $1 \mathrm{~s}$ are more common in Category $\mathrm{A}$, and 0 s are more common in Category B), and exemplars from different categories have fewer features in common.

Despite the fact that FR structures seem to characterize most real-world categories, people rarely provide FR sorts of stimuli like those in Table 1 in category construction experiments. Instead, people overwhelmingly sort on the basis of a single dimension, creating what is called a unidimensional sort (Ahn \& Medin, 1992; Medin et al., 1987). Using the items shown in Table 1, a unidimensional sort on the first dimension would put Exemplars 1, $3,4,5$, and 7 in one category and Exemplars 2, 6, 8, 9, and 10 in another category. When the exemplars are divided this way, the correlational feature structure is disrupted, limiting the inductive value of the categories. That is, Dimension 1 is predictable in each category, but the other features are not. Thus, it is puzzling why FR structure seems to be so common in real-world categories, but, when given the chance, people rarely sort on the basis of FR structure in experiments (see also Regehr \& Brooks, 1995).

Several researchers have discovered ways to facilitate FR category construction. Forcing subjects to process the relationships of different dimensions is one way to help subjects uncover the FR structure of experimenter-defined categories (Lassaline \& Murphy, 1996). Prior knowledge can also encourage FR sorting. Because prior knowledge provides explanations for many features, it also explains why groups of features occur together in some items. Using the bird example from above, knowing that something has wings supports the inference that it flies and therefore can nest on a cliff. Attempts to increase FR sorting by using features related to prior knowledge have met with moderate success (see Ahn, 1990; Medin et al., 1987; Spalding \& Murphy, 1996).

One limitation on the results of these prior experiments is that, in most studies, when knowledge was useful, it related all of the features of the category members. For example, Murphy and Allopenna (1994) and Spalding and Murphy (1996) used a category structure that had relevant features that were reliably associated with a category and irrelevant features that were random. When subjects had knowledge, all the relevant features were related to the theme, and none of the irrelevant features were. Thus, knowledge related all the critical features of 
the category. An analogous situation is true in a number of other prior experiments on knowledge effects (e.g., Ahn, 1990; Pazzani, 1991; Wattenmaker et al., 1986). Other experiments have not used all knowledge-based features (e.g., Wisniewski, 1995), but they have typically not then examined whether such features improve category learning or FR construction but instead compared features that were or were not related to the knowledge. However, the question of how knowledge influences category learning and category construction when the knowledge does not involve all of the features has not been addressed by these experiments.

In real life, prior knowledge does not always encompass every feature that people learn about a category. In general, any real category is probably a mixture of rote facts and characteristics that can be related to more general knowledge. For example, people may be able to explain how some of a bird's physical characteristics relate to its habitat and behaviors. However, their knowledge cannot explain every feature, such as why canaries are yellow and have short beaks but toucans are black and have large beaks. Undoubtedly, there are reasons behind these properties, but people can learn these facts and many others, without having ready explanations for them. Thus, many past experiments on prior knowledge have been somewhat unrealistic in their implicit assumption that all features would be connected by the knowledge.

Kaplan and Murphy (1998) examined the use of prior knowledge in supervised category learning when most of the new category's features were not related to prior knowledge. They used the same category structure as was used in the present experiments (see below for more detail). In this structure, each exemplar had only one thematic (knowledge-relevant) feature and five nonthematic (knowledge-irrelevant) features. Even this small amount of knowledge-related information influenced the ease with which subjects learned categories.

It is not yet known whether a small amount of background knowledge will facilitate FR category construction. This is an important question for two reasons. First, this way of learning categories is quite common. For example, Mervis (1987) suggests that children have already acquired the concepts behind many words on their own (i.e., without tuition) before they learn the word (see Merriman, Schuster, \& Hager, 1991; Quinn \& Eimas, 1997, p. 284). Indeed, studies of infant categorization almost always use an unsupervised learning task (e.g., Quinn \& Eimas, 1996; Younger \& Cohen, 1985). Thus, it is important to understand how prior knowledge influences this way of acquiring concepts, which apparently occurs across the lifespan.

The second reason to ask this question is that there is reason to believe that background knowledge must be very strong to help category construction. Spalding and Murphy (1996) showed that consistency of prior knowledge is important in this task: People rarely produce FR sorts when there are contradictions to their knowledge (yet contradictions do not stop knowledge from helping in a category learning task; Murphy \& Kaplan, 1998). Although we did not test contradictions in the present experiments, we did test situations in which the amount of knowledge-relevant information was minimal. Can people integrate sparse fragments of prior knowledge without the kind of direct support that characterizes supervised learning conditions? If the answer is yes, then knowledge will prove to be a powerful learning tool that does not require feedback to aid integration of features. However, since the dominant response in category construction is to focus on a single stimulus dimension, it is certainly possible that subjects will not be sensitive to a small amount of knowledge present in a different dimension. Unsupervised learning thus provides a stricter test of prior knowledge effects in category acquisition.

In the present experiments, we tested whether a minimal amount of prior knowledge-one feature per exemplar-affects the acquisition of the category structure. Specifically, we compared conditions in which the knowledge-relevant features were structurally consistent or inconsistent with the majority of the category's features, the knowledge-unrelated characteristic features. In Experiment 1 , we addressed the empirical question of whether our minimal knowledge structure can support the formation of FR categories. We examined whether our manipulation of the relationship between knowledge-relevant and knowledge-irrelevant features influences category construction, which is the usual dependent measure in unsupervised learning tasks.

Because these categories have a minority of features that are related to knowledge, they allowed us to investigate another important question of category acquisition-namely the relations between features that are tied to knowledge and those that are not. It has been suggested that when subjects use a theme to identify a category, they may not learn much about the other, nonthematic features-at least, when the theme is sufficient to classify category members (Murphy \& Allopenna, 1994). In Experiment 2, we investigated whether subjects notice the structure that relates the nonthematic features, both when there is a consistent theme and when there is not. In Experiments 3 and 4, we investigated whether subjects learn the relation between thematic features and nonthematic features. These experiments addressed whether the knowledge-relevant features stand alone as salient cues to categorization or become conceptually integrated with the rest of the category's features. Because subjects had not been taught categories, we could not use the normal classification task used in most experiments. Instead, in Experiments 3 and 4, we used a feature prediction task, in which one feature was provided, and subjects had to decide which of two alternatives would most likely occur with it. Subjects could perform above chance on this task only if they had learned which features co-occur in the 
learning set - that is, if they had extracted the feature clusters underlying the categories. This paradigm went beyond the usual category construction dependent measure by allowing us to investigate feature relations in a more detailed manner.

\section{EXPERIMENT 1}

Category construction tasks provide less support than standard category learning. In category learning, mistakes are corrected and subjects get several chances to classify the exemplars. Category construction is a more unconstrained task in which the subject has only one opportunity to make whatever categories he or she chooses, without feedback. In Experiment 1, we investigated whether a minimal amount of thematic information is sufficient to encourage subjects to construct FR categories, adopting the same dependent measure used in most other studies of category formation (Ahn, 1990; Lassaline \& Murphy, 1996; Medin et al., 1987; Spalding \& Murphy, 1996). Subjects viewed exemplars on a computer screen and then were asked to sort cards containing the exemplars into two groups in whatever way seemed best or most natural. The category structure used here (and in Kaplan \& Murphy, 1998) differed somewhat from those used previously. Because this structure was used throughout the experiments reported in this article, we will describe it in detail here. In this structure, shown in Table 2, each exemplar was defined by its values on five characteristic dimensions plus one idiosyncratic feature, which was derived from a theme. We call these features idiosyncratic because they occurred only in a single item, whereas the characteristic features repeated across items. All features were brief descriptive phrases.

The features on the characteristic dimensions were derived from the same domain, such as vehicles, but they were not related to more specific themes. For example,

Table 2

Category Structure for the Present Experiments

\begin{tabular}{|c|c|c|c|c|c|c|c|}
\hline \multirow[b]{3}{*}{ Exemplar } & \multicolumn{7}{|c|}{ Feature Type } \\
\hline & \multicolumn{5}{|c|}{ Characteristic } & \multicolumn{2}{|c|}{ Idiosyncratic } \\
\hline & D1 & D2 & D3 & D4 & D5 & Intact Theme & Mixed Theme \\
\hline \multicolumn{8}{|c|}{ Category A } \\
\hline $\mathrm{Al}$ & 1 & 1 & 1 & 1 & 1 & al & al \\
\hline $\mathrm{A} 2$ & 0 & 1 & 1 & 1 & 1 & $\mathrm{a} 2$ & $\mathrm{a} 2$ \\
\hline A3 & 1 & 0 & 1 & 1 & 1 & a3 & a3 \\
\hline A4 & 1 & 1 & 0 & 1 & 1 & $\mathrm{a} 4$ & b4 \\
\hline A5 & 1 & 1 & 1 & 0 & 1 & a5 & b5 \\
\hline A6 & 1 & 1 & 1 & 1 & 0 & a6 & b6 \\
\hline \multicolumn{8}{|c|}{ Category B } \\
\hline B1 & 0 & 0 & 0 & 0 & 0 & b] & bl \\
\hline B2 & 1 & 0 & 0 & 0 & 0 & b2 & b2 \\
\hline B3 & 0 & 1 & 0 & 0 & 0 & b3 & b3 \\
\hline B4 & 0 & 0 & 1 & 0 & 0 & b4 & a4 \\
\hline B5 & 0 & 0 & 0 & 1 & 0 & b5 & a5 \\
\hline B6 & 0 & 0 & 0 & 0 & 1 & b6 & a6 \\
\hline
\end{tabular}

the characteristic vehicle features were (Category A/ Category B) "has manual transmission/has automatic transmission," "has radial tires/has nonradial tires," "has air bags/has automatic seat belts," "license plate in front/ license plate in back," and "has vinyl seat covers/has cloth seat covers." While these are all clearly features of vehicles, prior knowledge of vehicles does not explain why these particular features should co-occur within a category (e.g., why radial tires would tend to go with air bags, and why nonradial tires would go with automatic seat belts).

In contrast, the idiosyncratic features $(\mathrm{a} 1-\mathrm{a} 6$ and $\mathrm{b} 1-\mathrm{b} 6$ of Table 2) were derived from themes, such as "arctic vehicle" versus "tropical vehicle" (lowercase " $a$ " features were derived from the " $A$ " theme, and lowercase " $b$ " features were derived from the " $B$ " theme). Continuing with the vehicle example, the idiosyncratic features were $(\mathrm{a} / \mathrm{b})$ "white/green," "made in Norway/made in Africa," "drives on glaciers/drives in jungles," "has treads/has wheels," "used in mountain climbing/used on safaris," and "heavily insulated/lightly insulated." These thematic features were derived from items that had elicited prior knowledge effects in both category learning (Murphy \& Allopenna, 1994) and category construction (Spalding \& Murphy, 1996), although, in those experiments, these thematic features were the only features that were valid for the categories. In all of the present experiments, each exemplar contained only one of these thematic featuresthe rest were not derived from themes. Thus, the themes could be identified only by integrating the related idiosyncratic features across exemplars. That is, unlike in most previous experiments, there was little in any one exemplar to give subjects the idea that the categories differed according to theme. Subjects would be unlikely to study one item with the feature "has treads," say, and think, "Oh, these are arctic vehicles," since none of its other features were related to the arctic vehicle theme. Instead, subjects would have to link the idiosyncratic properties (which do not recur across exemplars) and notice the theme that connected them on different trials. However, after seeing enough of the idiosyncratic features, subjects might notice that these nonrepeating features are related to themes. Then, upon seeing the exemplar with "has treads," the subjects might think, "This might be in the same category as the one that goes on glaciers."

Three distributions of idiosyncratic features were compared in Experiment 1. In the intact-theme condition, all of the idiosyncratic features in a given category were derived from the same theme. Thus, each exemplar was described by its values on the five characteristic dimensions, plus the idiosyncratic feature in the "Idiosyncratic/IntactTheme" column in Table 2. Note that within Category A, all of the idiosyncratic features begin with a lowercase " $a$," indicating the " $\mathrm{A}$ " theme, whereas the idiosyncratic features of Category B all begin with a lowercase " $b$," indicating the " $\mathrm{B}$ " theme. In the mixed-theme condition, 
half of the idiosyncratic features within each category were derived from one theme, and half were derived from the contrasting theme. Thus, each exemplar was described by five characteristic features plus the idiosyncratic feature in the last column of Table 2. As seen in Table 2, Exemplars A1-A3 each had one Theme A idiosyncratic feature, but Exemplars A4-A6 each had one Theme B idiosyncratic feature. For example, the idiosyncratic features for one of the mixed-theme vehicle categories were "has treads," "used in mountain climbing," "made in Norway," "drives in jungles," "green," and "lightly insulated." The first three idiosyncratic features were derived from the arctic vehicle theme, and the last three were derived from the tropical vehicle theme.

The main difference between this category structure and ones used in the past (e.g., Medin et al., 1987) was the presence of the idiosyncratic features. In order to assess whether the addition of these nonrepeating features would affect category construction, apart from the effects of prior knowledge, a control condition (the notheme condition) was created in which the idiosyncratic features were not derived from themes. If there were something about this category structure that encourages FR category construction, then this should be found in the no-theme condition as well.

The question of interest, then, was whether prior knowledge can influence category construction even when the features are generally not connected to prior knowledge that relates them. As discussed earlier, category construction experiments using structures similar to this one have resulted in predominantly unidimensional sorts, at least when the features were not related to prior knowledge. Given that the present structure consisted mainly of nonthematic features, there was good reason to expect that unidimensional sorts would be prevalent here as well. However, if people integrate the knowledge-relevant idiosyncratic features, as they do in the standard category learning task, then different sorting patterns would be expected. First, consider the intact- and mixed-theme categories. If, after viewing the exemplars several times, subjects notice the themes underlying the idiosyncratic features, then they might sort the cards on the basis of the themes. In this sense, the themes could be considered as an additional dimension on which people could sort. For the intact-theme categories, such a sort would also preserve the FR structure of the characteristic, nonthematic features. However, if the mixed-theme categories were sorted according to themes, and not on the basis of the characteristic features, then the FR category structure would be severely disrupted. That is, the resultant categories would be predictive with respect to themes, but not with respect to the characteristic features, which far outnumber the thematic features. Thus, the mixedtheme condition pitted the theme against the FR category structure. Finally, we expected many unidimensional sorts in the no-theme condition because there were no themes competing with the rest of the structure, and struc- tures similar to this one have generally yielded unidimensional sorts in past research.

\section{Method}

Subjects. Fifty-four University of Illinois undergraduates participated in this experiment for class credit or pay.

Materials and Design. The stimuli for this experiment consisted of nine different category pairs. The categories were from the domains of animals, buildings, and vehicles. Three different thematic conditions were used in this experiment: intact theme, mixed theme, and no theme, as described above. The animal themes could be paraphrased as "predator" versus "prey," the building themes as "space building" versus "ocean building," and the vehicle themes as "arctic vehicle" versus "tropical vehicle." A complete list of materials appears in the Appendix. The experiment was a 3 (thematic condition) $\times 3$ (domain) between-subjects design. Each subject saw only one category pair from one of these conditions.

Procedure. The experiment consisted of a study phase and a sorting phase. During study, the subjects viewed individual exemplars on a computer screen. Each exemplar consisted of a list of six features centered on the screen. The subjects pressed the space bar to view an exemplar, and the exemplar remained on the screen for $10 \mathrm{sec}$. The subjects studied six blocks of exemplars, each of which consisted of all 12 exemplars ( 6 in each category) in a different random order. The order of features on each stimulus presentation was randomized. The subjects were told to study the items so that they could answer questions about them later. They were not told that the items they were viewing were members of two different categories.

After the study phase, the subjects were given a stack of 12 index cards, each of which contained one exemplar from the study phase. The subjects were told to read over the cards and then sort them into two groups in whatever way seemed best or most natural.

\section{Results}

The sorts created by subjects were classified as family resemblance (FR), thematic, unidimensional (UNI), and other. Sorts were scored as FR only if the exemplars were sorted into the two categories shown in Table 2. Sorts were scored as thematic if all of the idiosyncratic features in each pile of cards were derived from the same theme. Note that FR and thematic sorts were isomorphic for the intact-theme categories, whereas FR and thematic sorts resulted in two different partitionings of the mixedtheme exemplars. A sort was scored as unidimensional only if all exemplars in one group contained one value on some characteristic dimension, and all of the exemplars in the other group contained the opposite value on that dimension. Any sort that failed to meet one of these criteria was scored as other. The breakdown of sorts by thematic condition is shown in Table 3.

Table 3

Number of Sorts by Condition in Experiment 1

\begin{tabular}{lcccc} 
& \multicolumn{4}{c}{ Sort Type } \\
\cline { 2 - 5 } Condition & FR & Thematic & UNI & Other \\
\hline Intact theme & 6 & $(6)^{*}$ & 8 & 4 \\
Mixed theme & 0 & 2 & 5 & 11 \\
No theme & 0 & $-\dagger$ & 15 & 3 \\
\hline
\end{tabular}

*The FR and thematic sorts are identical in the intact-theme condition, so the 6 thematic sorts are the same 6 listed under FR. †Thematic sorts were not possible in the no-theme condition. 
The primary question of interest was how the different conditions compared with respect to FR sorting. As seen in Table 3, 6 out of the 18 intact-theme subjects created perfect FR sorts, but none of the subjects in the mixedtheme condition or the no-theme condition produced perfect FR sorts. Fisher's exact tests showed that the intact-theme subjects created more FR sorts than did either the mixed-theme subjects or the no-theme subjects $(p s<.05)$. To assess the degree to which the subjects' sorts approximated perfect FR sorts (since the above analysis required perfect FR sorting), we calculated the FR deviation score for each subject's sort. The FR deviation was simply the minimum number of cards that would have to be moved from one group to the other in order to achieve a perfect FR partitioning of the cards. A perfect FR sort had an FR deviation of 0 ; so the lower the FR deviation score, the closer the sort approximates an FR sort. The FR deviation scores were entered into a 3 $\times 3$ between-subjects analysis of variance (ANOVA) with thematic condition (intact theme, mixed theme, and no theme) and domain (animals, buildings, and vehicles) as factors. There was a significant main effect of thematic condition $[F(2,45)=11.34, p<.001]$. Tukey's HSD test $(p<.05)$ revealed that the mixed-theme subjects had significantly higher FR deviations $(M=3.5)$ than did both the intact-theme $(M=1.7)$ and the no-theme $(M=$ 2.2) subjects, but the difference between the latter two groups was not significant. (The latter effect was due to the high proportion of unidimensional sorts in the notheme condition, which had FR deviations of only 2.) There was also an interaction between thematic condition and domain $[F(4,45)=4.06, p<.01]$, reflecting the fact that the differences between thematic conditions within the animal domain were much smaller than in the building and vehicle domains. However, they were in the same direction in all three domains. ${ }^{1}$

For the intact- and mixed-theme conditions, we also examined the degree to which the subjects produced thematic sorts. (Obviously, this analysis could not be done with the no-theme categories.) For the intact-theme subjects, a perfect thematic sort was the same as a perfect FR sort. For the mixed-theme subjects, however, a perfect thematic sort had Cards A1, A2, A3, B4, B5, and B6 in one group and Cards B1, B2, B3, A4, A5, and A6 in the other (separating the $a$ and $b$ idiosyncratic features; see Table 2). Theme deviation scores (the minimum number of cards that would have to be moved to make a perfect thematic sort) were computed for intact- and mixedtheme subjects. The theme deviation scores were entered into an ANOVA with thematic condition (intact theme and mixed theme) and domain (animals, buildings, and vehicles) as factors. There was a main effect of thematic condition, whereby the intact-theme subjects had lower theme deviations $(M=1.7)$ than did the mixed-theme subjects $(M=3.4)[F(2,30)=10.0, p<.01]$. Thus, the intact-theme subjects' sorts more closely approximated thematic sorts than did the mixed-theme subjects' sorts, even though the two groups saw the identical thematic features. There were no effects of domain.

\section{Discussion}

In Experiment 1, the subjects were able to integrate the thematic information across different items and apply it to their category constructions just as in the standard category learning task (Kaplan \& Murphy, 1998). This shows that people can spontaneously exploit their prior knowledge in an unsupervised learning situation. Even small doses of knowledge-relevant information can influence learning without the benefit of feedback. The no-theme condition sorting pattern suggests that there is a unidimensional bias with this structure, as has consistently been found with similar structures when the features are not related to prior knowledge (Ahn \& Medin, 1992; Medin et al., 1987). When knowledge was added to this structure, the subjects were much less likely to create unidimensional sorts. However, the effects of prior knowledge on category construction varied depending on whether the knowledge was consistent with the FR category structure, as it was in the intact-theme condition, or orthogonal to the FR structure, as it was in the mixedtheme condition.

One third of the intact-theme subjects uncovered the intended FR categories, suggesting that a small amount of knowledge can help when it is consistent with the FR category structure. While $33 \%$ may not seem like a very large percentage, it is a remarkable effect considering the small manipulation: Only one of the six features in each exemplar was related to the theme. Also, $33 \%$ is considerably higher than the $0 \%$ FR sorting in both the mixedtheme condition and the no-theme condition.

The results from the mixed-theme condition suggest that even when the themes are uncorrelated with the FR category structure, prior knowledge still influences category construction. Whereas the thematic features in the intact-theme condition helped to make the FR structure more apparent, the inconsistency of the themes and the FR structure in the mixed-theme condition made FR detection rather difficult, as evidenced by the significant difference in their FR deviation scores. So, when prior knowledge conflicts with the FR structure, the FR structure will not be uncovered; but, by the same token, prior knowledge alone does not determine the sorts either. In fact, when the knowledge is orthogonal to the FR category structure, it seems to wreak havoc with subjects' sorts, as indicated by the large number of other sorts in the mixedtheme condition that did not obey thematic, FR, or even unidimensional constraints. If the mixed-theme subjects had simply ignored the knowledge-relevant features, they would have created predominantly unidimensional sorts, as did the no-theme subjects. If they had ignored the characteristic features (which would have meant ignoring most of the features), they would have created thematic sorts. Instead, they seemed to be influenced by both the thematic and the nonthematic features. The subjects 
were best at identifying the category structure when it coincided with knowledge structures-a conclusion that is consistent with experiments using rather different learning paradigms (Pazzani, 1991; Wattenmaker, 1995; Wattenmaker et al., 1986).

In Experiments 2 and 3, we attempted to uncover in more detail what subjects learned about the category structure when knowledge was helpful or unhelpful.

\section{EXPERIMENT 2}

Experiment 1 showed that people were more likely to create perfect FR sorts when the minimal amount of knowledge-relevant information present in the categories was perfectly correlated with the FR structure. However, a perfect FR sort does not necessarily mean that the characteristic feature correlations were learned. In the intacttheme categories, the thematic features were perfectly correlated with the underlying FR characteristic feature structure. So, if someone had sorted on the basis of the thematic features alone, the characteristic features would have ended up in an FR structure simply because of the correlation of structure and theme. Such a subject may never have noticed that some characteristic features tended to go together. Alternatively, it is possible that the subjects who created the FR sorts actually noticed the relationships between the characteristic features and took this into consideration while sorting. The results of Experiment 1 support this possibility, because they show that when the FR structure did not coincide with the theme (i.e., the mixed-theme condition), the subjects did not sort according to that structure-the FR structure and the theme had to pick out the same category structure in order for it to be recovered.

There is some evidence from the category learning paradigm (which involves labeled exemplars and constant feedback) that subjects taught intact-theme categories identical to the ones in the present experiments learn quite a bit about the characteristic features. Kaplan and Murphy (1998) had subjects make speeded single-feature classifications after learning either intact-theme or mixed-theme categories. In this test, single features appeared on the screen, and subjects had to decide as quickly and accurately as possible which category the feature usually appeared in. Despite the fact that intact-theme subjects learned the categories in about half as many blocks as did the mixed-theme subjects (and therefore had seen each of the features half as often), the intacttheme subjects were no slower or less accurate at classifying characteristic, nonthematic features than the mixed-theme subjects were. Thus, when the learning situation provides support, prior knowledge does not impede the learning of the knowledge-irrelevant features. What is not clear from that study is whether this kind of information will be acquired in an unsupervised learning situation. In category learning, all the features are paired with the category name (the feedback), whereas there is no name in the unsupervised case. Thus, it is certainly pos- sible for subjects in an unsupervised learning situation not to learn which nonthematic features go with which category.

In the present experiment, we used a feature inference task to test how well people had learned the feature correlations after just observing, and not categorizing, the exemplars. After viewing exemplars for six blocks, subjects completed a series of inference trials in which they were asked what value an object was more likely to have on some characteristic dimension (e.g., automatic or manual transmission), given a value on another characteristic dimension (e.g., cloth seat covers). Unsupervised category formation is driven by noticing feature correlations in the environment, so this feature inference task was chosen because it measures the degree to which such correlations are learned through observation alone (see also Billman \& Knutson, 1996). This task provided a way to gauge unsupervised category learning in more detail than the sorting task, because an item could be sorted on the basis of any one of its six features, but the inference task systematically tested all features. Furthermore, this experiment provide an important advance over Kaplan and Murphy's (1998) learning experiment, because, in the present experiment, all subjects saw the items for the same number of trials. (In learning experiments, subjects typically study the items until they reach a criterion, usually resulting in unequal numbers of stimulus exposures across conditions.) Thus, sensitivity to category structure could be compared more fairly here.

In the present category structure, the characteristic feature correlations were identical for the intact-theme and mixed-theme categories. Therefore, inferences between characteristic features allowed us to test how the distribution of thematic features affects what is learned about the category structure, as represented by the nonthematic features: Will people learn the nonthematic feature structure better if the thematic features are correlated rather than uncorrelated with this underlying structure?

\section{Method}

Subjects. Thirty-six University of Illinois undergraduates participated in this experiment for class credit.

Procedure. The same intact- and mixed-theme categories used in Experiment 1 were used here. The no-theme categories were not used in this experiment because the question of interest was how the themes affected the learning of the characteristic features. This experiment consisted of two parts, a study phase and an inference phase. The study phase was identical to that of Experiment 1 . After study, the subjects completed 20 inference trials. In the inference trials, the subjects were told that an object had a certain feature on one dimension, and they had to decide which of two features the object was most likely to have on another dimension. For example, one inference asked, "Suppose an animal has the feature: 'Spotted.' Which of the features below is it more likely to have: 'Has a short snout,' or 'Has a long snout?" "The given feature was written in the middle of the screen, and the option features were written in the lower left and right corners of the screen. The subjects pressed the " $Z$ " key to indicate the left-hand choice and the "/" key to indicate the right-hand choice. The subjects were instructed to base their judgments only on the exemplars in the experiment and not on objects they knew about in the real world. 
The 10 critical inferences were characteristic-to-characteristic feature inferences, in which the subjects were given a feature on a characteristic dimension and asked about the value of a different characteristic dimension. Formally (see Table 2), if the given feature had the value 1 , then the correct answer was 1 on the other dimension. If the given feature had the value 0 , then the correct answer was 0 on the other dimension. Each of the 10 characteristic features appeared as the given feature once, and each of the five characteristic dimensions appeared twice as options. Each of the 10 characteristic-to-characteristic inferences asked about a unique pair of dimensions. All of these questions and their correct answers were identical for the intact-theme and mixed-theme categories, because the categories had identical characteristic features. The rest of the inferences involved both characteristic and idiosyncratic (thematic) features. Because the statistical relationships between the themes and characteristic features were not the same for the mixed-theme and intact-theme conditions, differences on these inferences between the two conditions could not be meaningfully compared, and so they are not reported. The 20 inference questions were randomly ordered for every subject.

\section{Results}

The main question of interest was whether the intacttheme and mixed-theme groups learned the characteristicto-characteristic feature correlations to the same degree. The number of correct responses out of 10 for each subject was entered into an ANOVA with thematic condition (intact theme vs. mixed theme) and domain (animals, buildings, and vehicles) as factors. There was a main effect of thematic condition whereby the intact-theme subjects were more accurate $(M=6.4)$ than mixed-theme subjects $(M=5.0$, which was chance) on the inferences $[F(1,30)=7.79, p<.01]$. Thus, it appears that people learn more about the characteristic feature structure when the small amount of knowledge is correlated with the structure. There was also a main effect of domain $[F(2,30)=3.61, p<.05]$, which was driven by the fact that accuracy was significantly lower for animals $(M=$ 4.8) than for vehicles $(M=6.3)$ (see note 1). Inspection of our data showed that, in all experiments reported here, effects were not driven by one or two particularly easy or difficult feature inferences. More detailed examination of specific items was not possible, due to the low number of data points per item. ${ }^{2}$

\section{Discussion}

Experiment 2 showed that even a little bit of knowledge facilitated the learning of the category structure when the knowledge was consistent with that structure. The subjects learned the characteristic feature correlations better when the knowledge-relevant features were correlated with the characteristic features than when they were orthogonal to the characteristic features. This result - that the consistent knowledge made the characteristic feature structure easier to learn, even though the knowledge was not embodied in the characteristic features-is surprising. It shows that the role of prior knowledge is not just to facilitate the learning of the features that can be directly related to it. Rather, people are sensitive to the features that tend to co-occur with the knowledge- relevant properties; identifying the knowledge structure helps in identifying the empirical structure.

Noticing the feature correlations is tantamount to learning the category in an unsupervised learning situation. Experiment 2 demonstrated that knowledge highlights the feature structure-somehow it unites the exemplars and allows subjects to recognize the typical feature clusters in the structure. In the real world, prior knowledge may allow people to detect clusters of "rote fact" features if these features happen to co-occur with the features that they can explain with prior theories. To return to the example from the introduction, if we notice that several different animals have wings, fly, and live in trees, we may also notice that these same animals tend to share a particular beak shape, color, and song. Once several individual objects can be united through background knowledge, people may begin to notice that these items share a host of other features as well. Thus, the effects of prior knowledge on unsupervised category learning extend beyond the learning of the knowledge-relevant features.

\section{EXPERIMENT 3}

The intriguing result of Experiment 2 raises the question of how knowledge has this effect on the acquisition of features that are not directly related to it. One explanation is that the knowledge-relevant idiosyncratic features were simply salient cues, much like category labels, that highlighted the underlying category structure. For example, it is possible that each individual (idiosyncratic) arctic vehicle feature tagged the entire exemplar as something different from exemplars containing a single (idiosyncratic) tropical vehicle feature. On this account, the idiosyncratic features can be seen as instantiating an explicit "theme" dimension, where all arctic vehicle features had one value (i.e., 0 ) and all tropical vehicle features had the other value (i.e., 1). If this were the case, each idiosyncratic feature would essentially be a label for one category or the other. Because the knowledge-related features were orthogonal to the FR structure in the mixedtheme categories, this salient cue would not highlight the FR structure - in fact, it would hinder the acquisition of the structure. In short, this account says that the effect of knowledge is to provide a salient cue that creates a structure used to organize the other features.

An alternative account is that knowledge becomes more actively integrated with the other features, which facilitates acquisition of the FR structure. For example, if "vinyl seat covers" is a characteristic feature that usually occurs with the tropical vehicle exemplars, then subjects might begin to think that vinyl seat covers are especially suited for warm weather vehicles (Kaplan \& Murphy, 1998, mention anecdotal evidence for this phenomenon).

If this cuing account is true, then an equally salient cue, related to the category structure in the same way that the knowledge-relevant features were before, should 
have the same effect. Knowledge connections per se should not be necessary. In Experiment 3, color was used as the salient cue: Each of the exemplars was written in a different shade of either red or blue. In the consistent condition (analogous to the intact-theme condition), the color was correlated with the FR structure. In the inconsistent condition (analogous to the mixed-theme condition), color was orthogonal to the FR category structure. If the salient cue hypothesis is correct, then the consistent color group should perform better on characteristic inferences than the inconsistent color group. Of course, coloring the entire exemplar is quite different from providing a single featural cue with each exemplar. However, we expected any cuing effect to be greater here than in Experiment 2, given that color is a perceptually striking cue that is immediately apparent in viewing each item (unlike the theme, which had to be abstracted across exemplars) and is present in every feature of an item. Although subjects who are paying little attention might well not notice a theme, even the least diligent subject should notice the color difference.

\section{Method}

Subjects. Thirty University of Illinois undergraduates participated in this experiment for class credit or pay.

Materials and Design. The categories used in this experiment were formally identical to the categories used in Experiment 2, with the exception that the present categories had no idiosyncratic, thematic features. Thus, each exemplar consisted of a list of five characteristic, nonthematic features. In place of idiosyncratic features, the features of each exemplar were written in a unique shade of either blue or red. Six of the exemplars were red, and six were blue (all features within a single exemplar were the same color). Formally, the lowercase "a"s in Table 2 were replaced with six different shades of red, and the lowercase " $b$ "s were replaced with six different shades of blue. (Unique shades were used because the thematic features used in past experiments were also unique, even though they were derived from one of the two themes.) In the consistent condition, the colors were correlated with the FR feature structure, just as the themes were correlated with the FR structure in the intact-theme condition above. In the inconsistent condition, three of the exemplars in each category were blue and three were red, just as the themes were uncorrelated with the FR structure in the mixed-theme categories before (as shown in Table 2).

Procedure. The experiment consisted of a study phase and an inference phase, as in the previous experiments. During study, the subjects saw six blocks of exemplars in a random order. In the inference phase, the subjects answered 20 characteristic-to-characteristic inferences. (Obviously, no thematic inferences were possible.) Each feature occurred once as the given feature, and each subject saw all possible combinations of dimensions in the inference trials. During the inference phase, all of the features were written in black.

\section{Results and Discussion}

The inference accuracies were calculated for each subject and entered into a two-way ANOVA with color condition (consistent vs. inconsistent) and domain (animals, buildings, and vehicles) as factors. There were no significant main effects or interactions. There was no significant difference between the accuracy of the consistent subjects $(M=62 \%)$ and the inconsistent subjects
$(M=66 \%)$. Both were significantly better than chance [for the consistent group, $t(14)=2.72, p<.05$; for the inconsistent group, $t(14)=4.02, p<.05$ ]. Thus, unlike knowledge, color does not make the FR structure any clearer when the color is correlated with the characteristic features than when the color is orthogonal to the FR structure. Indeed, the effect is in the opposite direction from the effect of theme in Experiment 2. This shows that the knowledge-relevant idiosyncratic features are not serving as simple category tags. Knowledge appears to affect the acquisition of the characteristic features in a different manner than a salient cue does.

Interestingly, although there was no significant difference between the consistent and inconsistent groups, both appear to have learned considerably more than the mixed-theme group in Experiment 2. One possible explanation for this is that, without the 12 idiosyncratic features, there was less noise obstructing the characteristic feature correlations. In addition, the inconsistency of the themes with the FR structure in the mixed-theme categories might have disrupted the detection of the feature correlations. That is, if mixed-theme subjects noticed the themes and tried to recruit the characteristic features into the themes, they would be faced with a set of feature correlations different from the ones underlying the FR categories. Finally, it is possible that subjects found the colored exemplars more interesting to look at for six blocks, and so they paid more attention to the exemplars than did the subjects in Experiments 1 and 2. In any case, it is clear that providing a cue to category structure by itself does not improve subjects' ability to acquire that structure in an unsupervised learning situation.

\section{EXPERIMENT 4}

Experiments 1 and 2 showed that subjects observing intact-theme categories learned quite a bit about the underlying FR feature structure. Similarly, in the real world, people learn some features that can be related to prior knowledge (e.g., why wings allow flying and nesting in trees) along with those "rote" features that cannot be explained by prior knowledge (e.g., that robins have red breasts). In Experiment 4 , we asked whether people relate the nonthematic,

Table 4

Structure of Category A for Experiment 4, Predatory Animals Feature Type

\begin{tabular}{ccccccl} 
& \multicolumn{5}{c}{ Characteristic } & \multicolumn{1}{c}{ Idiosyncratic } \\
\cline { 2 - 6 } Exemplar & Snout & D2 & D3 & D4 & D5 & \multicolumn{1}{c}{ Id be tomesticated } \\
\hline A1 & long & 1 & 1 & 1 & 1 & Can't be dome \\
A2 & short & 1 & 1 & 1 & 1 & Eats meat \\
A3 & long & 0 & 1 & 1 & 1 & Has sharp teeth \\
A4 & long & 1 & 0 & 1 & 1 & Aggressive \\
A5 & long & 1 & 1 & 0 & 1 & Lives alone \\
A6 & long & 1 & 1 & 1 & 0 & Has a barbed tail \\
\hline
\end{tabular}

Note-Dimension 1 (D1) was snout length; the other dimensions (D2-D5) are listed schematically. 
characteristic features to the thematic, knowledge-based features. As we mentioned earlier, one explanation of how the themes improved category formation and characteristic-to-characteristic feature inferences is that the features were related to the knowledge structures in some way. Even though each thematic feature occurred in only one item, it is possible that the subjects identified the theme as a whole (e.g., "some kind of predatory animal") and then attempted to connect the characteristic features to this theme (e.g., "maybe the long snout helps it locate prey better"). In Experiment 4, we tested this hypothesis by asking whether subjects learned feature-to-feature relations that were consistent with such knowledge generalizations better than those that were inconsistent.

To explain the particular comparison we made, examine the sample category shown in Table 4 . This represents the predatory animal category in the intact-theme condition. (Because only the intact-theme categories were shown to facilitate unsupervised FR category learning as measured by category construction and feature inferences, only intact-theme categories were used in this experiment.) We have filled in the exact idiosyncratic features used here, as well as one of the characteristic dimensions (rather than using 1s and 0s), in order to aid exposition.

When subjects receive examples of this sort, mixed up with prey animals, they may notice that some of the items have predatory features and some have prey features. Furthermore, as described above, they may notice that the predatory animals tend to have a long snout (though A2 does not), perhaps to aid in locating prey. If subjects form these kinds of generalizations between characteristic and thematic features, they should find it easier to make a feature-to-feature inference when it is consistent with that generalization.

Consider, for example, the question, "If an animal has sharp teeth, does it have a long or a short snout?" As Table 4 shows, the item that has sharp teeth also has a long snout. Furthermore, predatory animals in general have long snouts, so even if the subject does not recall this particular exemplar, he or she can provide the right answer based on this generalization (because sharp teeth are associated to the predatory animal theme). In contrast, consider the question, "If an animal eats meat, does it have a long or a short snout?" As Table 4 reveals, the item that eats meat has a short snout-but this is inconsistent with the generalization that predatory animals have long snouts. Thus, if subjects are learning and using these generalizations of the relation between thematic and characteristic features, they should find the correct answer to this question more difficult to give. In Experiment 4 , we performed this kind of contrast, between questions that were consistent or inconsistent with the overall feature-theme correlations, to discover whether subjects had learned such correlations between thematic and characteristic features. In addition to the questions asking subjects to predict characteristic features from the- matic features (like the above examples), we asked the reverse kind of question (e.g., "If an animal has a short snout, would it be more likely to eat meat or plants?").

\section{Method}

Subjects. Thirty University of Illinois undergraduates participated in this experiment for class credit.

Materials and Procedure. The same intact-theme categories that were used in Experiments 1 and 2 were used in this experiment. As before, the subjects studied six blocks of exemplars from one of the domains of animals, buildings, or vehicles. In the inference phase, the subjects answered 20 inferences, 10 of which were consistent and 10 of which were inconsistent with the usual relations between characteristic features and the themes, as described above. The characteristic-to-characteristic inferences used previously were not tested here.

\section{Results and Discussion}

The subjects' accuracies on the two types of inferences were entered into a repeated measures ANOVA with domain (animals, buildings, and vehicles) as a betweensubjects factor and inference type (consistent and inconsistent) as a within-subjects factor. The subjects were significantly more accurate on consistent inferences $(M=62 \%)$ than on inconsistent inferences $(M=50 \%$, which was chance) $[F(2,33)=8.26, p<.01]$. No other effects were significant.

This result suggests that the subjects might have formed prototypes of the categories that captured both the thematic and the characteristic features. Such a prototype would include the typical characteristic features on each characteristic dimension plus the idiosyncratic features consistent with one theme. Using the predatory animal example, this prototype would include some of the characteristic features, "has a long snout," "spotted," "has pointed ears," "can swim," and "active at night," plus the theme, which could be activated by any of the features, "has sharp teeth," "lives alone," "eats meat," "has a barbed tail," "aggressive," and "can't be domesticated." Then, when any one of the thematic features activated the theme, the typical characteristic features that were learned would be activated as well. So, when the subjects were asked which nonthematic characteristic feature belonged with a certain thematic feature, they might have accessed information from the prototype instead of an individual exemplar. Consequently, the subjects were relatively accurate on consistent inferences because the prototypes contained the correct characteristic-idiosyncratic correlations. When the inferences involved atypical features, however, the prototype for the wrong theme was activated, causing the subjects to make more errors on these inferences. In sum, consistent prior knowledge not only highlights the relationships between the nonthematic features, but it also links together the thematic and nonthematic features. This could occur if subjects attempt to incorporate the characteristic features directly into the theme. That is, subjects might interpret a nonthematic feature as being related to the theme (e.g., predatory an- 
imals might have pointed ears to help them listen for their prey).

Heit $(1994,1998)$ has shown that prior knowledge influences people's judgments about pairs of features in questions that were similar to the ones asked here. However, his stimuli were quite different (among other things, they were all in one category, rather than representing two different categories that subjects might separate), and he did not include the neutral "rote" features considered here. For example, Heit showed that subjects were influenced by their prior knowledge to rate properties such as "shy" and "does not attend parties often" as cooccurring more often than pairs such as "shy" and "attends parties often." The present experiment extends such findings by showing that subjects make connections between knowledge-related features and those that are not connected by knowledge (prior to the experiment), when the features are all associated with the same category.

\section{GENERAL DISCUSSION}

The experiments reported here clearly showed that a minimal amount of knowledge-relevant information facilitates unsupervised FR category learning. Experiment 1 showed that people were more likely to create perfect FR sorts when the little bit of thematic information in the categories was consistent with the underlying FR structure than when the same amount of knowledge was uncorrelated with the FR structure. Perhaps the more surprising result was that, in the later experiments, consistent prior knowledge facilitated the learning of much of the category and not just the features that could be easily related to the knowledge. Experiment 2 showed that people learned significantly more about the characteristic, nonthematic features when the little bit of knowledge was correlated with those features (as in the intact-theme categories) than when the knowledge-relevant features were orthogonal to the FR structure (as in the mixed-theme categories). Experiment 4 showed that generalizations about the characteristic features were tied to the themes as well. Overall, Experiments 2 and 4 highlighted the fact that consistent prior knowledge facilitates learning about all features of the category, and not just the ones that can be directly related to the knowledge. Wattenmaker (1991) and Billman and Knutson (1996) showed that feature correlations can be learned from simple exposure to exemplars without explicit category instruction (but see Anderson \& Finchman, 1996, for a conflicting result with pictorial, continuous dimensions), but they did not manipulate prior knowledge. The present experiments revealed that this ability can be mediated by prior knowledge. If the FR structure is consistent with the knowledge, people learn a great deal about the category. However, when the same amount of knowledge is inconsistent with the FR category structure, feature learning is disrupted.

Taken together, these results extend the positive effects of prior knowledge to learning contexts in which the categories contain a minimal amount of knowledgerelevant information and in which the learning task provides no support. Despite the similar advantages of prior knowledge in supervised and unsupervised learning situations, inconsistent prior knowledge seems to hurt learning more in unsupervised learning tasks than in supervised learning tasks. In Experiment 1, the mixed-theme subjects were significantly worse than the no-theme subjects with respect to FR sorting. In contrast, there was no difference between the corresponding conditions in the standard category learning task (Kaplan \& Murphy, 1998). This suggests that inconsistent prior knowledge is more harmful when feedback is unavailable. In supervised category learning, people can quickly learn to ignore the themes as cues to categorization when the themes are unhelpful, because the feedback contradicts the thematic cues. However, in category construction, the inconsistency of the themes and the FR structure obscures the underlying structure, and, in this task, there are no signals from a teacher reinforcing the structure. So, while the mixed-theme categories lead to an incorrect first impression of the stimuli, feedback in supervised category learning provides a second chance at deriving the underlying structure, but unsupervised category construction does not.

Given the tendency for subjects to create unidimensional sorts when faced with the no-theme categories, some researchers (Ahn \& Medin, 1992; Wisniewski \& Medin, 1994) have argued that a thematic sort is actually a unidimensional sort on some higher level thematic dimension. This possibility does not seem consistent with the finding that the intact-theme subjects appear to have learned a great deal about a number of the features of the categories-not just the ones that would be involved in the higher level dimension. This somewhat surprising result suggests that when the knowledge is consistent with the structure of the whole category, people learn more about several of the features in the category, rather than simply learning the theme itself (as was suggested by Murphy \& Allopenna, 1994). Although subjects are clearly noticing that some of the items are underwater buildings, say, they do not stop at this but instead use this knowledge in learning the correlations of other features.

Indeed, the critical question for future research is how knowledge influences the learning of feature correlations. One possibility is that knowledge might imply to people that the characteristic features are not random. If knowledge can explain the distribution of some of the features (namely, the thematic features), then people might believe that there are also principled reasons for the clusters of the characteristic features as well. That is, if people detect that part of the structure is coherent, perhaps they will believe that the organization of the remaining features is not accidental. This idea is related to the notion that people behave as if many objects have essences that give rise to their features (Medin \& Ortony, 1989). Another possibility is that knowledge simplifies learning 
by reducing the number of feature correlations that have to be learned. Once subjects identify the themes and learn that some characteristic feature tends to go with one theme (e.g., arctic vehicles tend to have four doors), they can immediately deduce that the contrasting characteristic feature goes with the other theme (e.g., the tropical vehicle has two doors). When the themes are not correlated with the FR structure (or when the themes are absent), subjects are faced with a large number of possible relations of features: Each characteristic feature has a correlation with 10 other features within its category (4 characteristic and 6 idiosyncratic, as shown in Table 1). By reducing the idiosyncratic features to their themes and relating each characteristic feature to one of two themes, the number of correlations that have to be learned is greatly reduced. Although knowledge may have such an effect, it cannot be the entire story, because the substitution of an equally predictive, but not thematic, consistent cue did not help learning differentially for the consistent over inconsistent conditions (Experiment 3), even though it was perceptually much more salient.

These results are consistent with a finding by Wisniewski and Medin (1994) that prior knowledge and data interact in category learning. In their experiments, subjects were shown a series of children's drawings and were asked to derive a rule that would divide the drawings into two groups. In the theory condition, subjects were told that the pictures were drawn by two different kinds of children (e.g., creative children vs. noncreative children). Control subjects were simply told that the drawings were done by children in Group A and Group B. Subjects in the theory condition devised hierarchical rules that incorporated features from the drawings into their theories about the children who drew them. Hierarchical rules take a concrete feature and use it to support a more abstract dimension that relates to the theory. For instance, a collar (concrete feature) might be taken as evidence of detail (abstract dimension), indicating that the drawing was done by a creative child (the theory that creative children make more detailed pictures). In Wisniewski and Medin's experiment, category knowledge affected how the features were used in the rules. Subjects in the theory condition used hierarchical rules, such as the one outlined above, $63 \%$ of the time. In contrast, control subjects used such rules only $22 \%$ of the time; they preferred concrete features. For example, subjects without the background knowledge tended to mention a specific feature of the clothing in the drawing (e.g., a collar) without relating that feature to a more abstract dimension.

Similarly, in the present experiments, knowledge may have prompted the subjects to search for ways to connect the nonthematic features with the thematic ones. The nonthematic features could have been recoded or related through hierarchical structures primed by the knowledge, resulting in feature chains similar to those used by Wisniewski and Medin's (1994) subjects. For example, on noticing that predatory animals are active at night, one might infer that being active at night is an advantage for a predatory animal because it could surprise its sleeping prey. Thus, knowledge could have acted to assimilate the characteristic features once the basic knowledge structures were identified in the stimuli, which in turn would have aided learning of the category structure. Experiment 4 showed that subjects seem to have formed generalizations between the knowledge-related and knowledgeunrelated features, such as "the predators have long snouts," providing evidence for this proposal.

In conclusion, the experiments reported here broaden the scope of prior knowledge effects in category learning by showing that a minimal amount of consistent knowledge aids in identifying FR category structure. Importantly, the effects of knowledge were not limited to the few features that carried the knowledge. Rather, consistent prior knowledge enhanced the learning of several of the category's nonthematic features. In addition, subjects spontaneously accessed their knowledge in the absence of hints or even feedback, driven only by the objects' properties. Throughout these experiments, people capitalized on very sparse associations to their knowledge, showing that knowledge can be a powerful organizing principle, allowing them to notice seemingly arbitrary clusters of features. Thus, knowledge can be viewed as a foundation, which can support learning of empirical structure.

To end with a speculative point, we return to our earlier observation that much category learning in infancy and early childhood may be through unsupervised learning (see Quinn \& Eimas, 1997, p. 284, for a discussion of the theoretical importance of this claim). One puzzle about the knowledge-based approach to concepts (e.g., Murphy \& Medin, 1985) is how background knowledge can influence early concept learning when young children are unlikely to have anything like adult knowledge of biology, physics, social conventions, and so on. The present experiments are important in showing that even a very small amount of knowledge is helpful in unsupervised learning. So, for example, if infants were to know nothing about animals except that they use their limbs to locomote (a primitive kind of background knowledge), this might still be helpful in learning categories of animals in an unsupervised learning task (e.g., BehlChadha, 1996), just as the knowledge connecting one feature per exemplar was helpful in our task. Our results show that complete theories of a domain are not necessary to achieve the benefits of knowledge, and so knowledge effects may extend into quite early childhood, where knowledge is sketchiest and least complete.

\section{REFERENCES}

AHN, W. (1990). Effects of background knowledge on family resemblance sorting. Proceedings of the 12th Annual Conference of the Cognitive Science Society (pp. 149-156). Hillsdale, NJ: Erlbaum.

AHN, W., \& MEDIN, D. L. (1992). A two-stage model of category construction. Cognitive Science, 16, 81-121.

Anderson, J. R., \& Finchman, J. M. (1996). Categorization and sensitivity to correlation. Journal of Experimental Psychology: Learning, Memory, \& Cognition, 22, 259-277.

BeHL-ChADHA, G. (1996). Basic-level and superordinate-like categorical representations in early infancy. Cognition, 60, 105-141.

Billman, D., \& KNutson, J. (1996). Unsupervised concept learning 
and value systematicity: A complex whole aids learning the parts Journal of Experimental Psychology: Learning, Memory, \& Cognition, 22, 458-475.

CAREY, S. (1985). Conceptual change in childhood. Cambridge, MA: MIT Press.

HEIT, E. (1994). Models of the effects of prior knowledge on category learning. Journal of Experimental Psychology: Learning, Memory, \& Cognition, 20, 1264-1282.

HErT, E. (1998). Influences of prior knowledge on selective weighting of category members. Journal of Experimental Psychology: Learning, Memory, \& Cognition, 24, 712-731.

KAPLAN, A. S., \& MURPHY, G. L. (1998). Interactions of structure and knowledge in family resemblance category learning. Manuscript submitted for publication.

KeIL, F. C. (1989). Concepts, kinds, and cognitive development. Cambridge, MA: MIT Press.

LASSALINE, M. E., \& MURPHY, G. L. (1996). Induction and category coherence. Psychonomic Bulletin \& Review, 3, 95-99.

Medin, D. L., \& Ortony, A. (1989). Psychological essentialism. In S. Vosniadou \& A. Ortony (Eds.), Similarity and analogical reasoning (pp. 179-195). New York: Cambridge University Press.

Medin, D. L., Wattenmaker, W. D., \& Hampson, S. E. (1987). Family resemblance, conceptual cohesiveness, and category construction. Cognitive Psychology, 19, 242-279.

Merriman, W. E., Schuster, J. M., \& Hager, L. (1991). Are names ever mapped onto preexisting categories? Journal of Experimental Psychology: General, 120, 288-300.

Mervis, C. B. (1987). Child-basic object categories and early lexical development. In U. Neisser (Ed.), Concepts and conceptual development: Ecological and intellectual factors in categorization (pp. 201233). New York: Cambridge University Press.

MURPHY, G. L. (1993). Theories and concept formation. In I. V. Mechelen, J. Hampton, R. Michalski, \& P. Theuns (Eds.), Categories and concepts: Theoretical views and inductive data analysis (pp. 173200). London: Academic Press.

MurPhy, G. L., \& AllopenNA, P. D. (1994). The locus of knowledge effects in concept learning. Journal of Experimental Psychology: Learning, Memory, \& Cognition, 20, 904-919.

MurPhy, G. L., \& KaPLAN, A. S. (1998). Feature distribution and background knowledge in category learning. Manuscript submitted for publication.

MurPhy, G. L., \& Medin, D. L. (1985). The role of theories in conceptual coherence. Psychological Review, 92, 289-316.

PAZZANI, M. J. (1991). Influence of prior knowledge on concept acquisition: Experimental and computational results. Journal of Experimental Psychology: Learning, Memory, \& Cognition, 17, 416-432.

QuinN, P. C., \& Eimas, P. D. (1996). Perceptual cues that permit categorical differentiation of animal species by infants. Journal of Experimental Child Psychology, 63, 189-211.
QuinN, P. C., \& Eimas, P. D. (1997). A reexamination of the perceptualto-conceptual shift in mental representations. Review of General Psychology, 1, 271-287.

Regehr, G., \& Brooks, L. R. (1995). Category organization in free classification: The organizing effect of an array of stimuli. Journal of Experimental Psychology: Learning, Memory, \& Cognition, 21, 347. 363.

Rosch, E., \& Mervis, C. B. (1975). Family resemblance: Studies in the internal structure of categories. Cognitive Psychology, 7, 573605.

Smith, E. E., \& Medin, D. L. (1981). Categories and concepts. Cambridge, MA: Harvard University Press.

SPalding, T. L., \& MurPhy, G. L. (1996). Effects of background knowledge on category construction. Journal of Experimental Psychology: Learning, Memory, \& Cognition, 22, 525-538.

WATTENMAKER, W. D. (1991). Learning modes, feature correlations, and meinory-based categorization. Journal of Experimental Psychology: Learning, Memory, \& Cognition, 17, 908-923.

WATTENMAKER, W. D. (1995). Knowledge structures and linear separability: Integrating information in object and social categorization. Cognitive Psychology, 28, 274-328.

Wattenmaker, W. D., Dewey, G. I., Murphy, T. D., \& Medin, D. L. (1986). Linear separability and concept learning: Context, relational properties, and concept naturalness. Cognitive Psychology, 18, 158-194.

WISNIEWSKI, E. J. (1995). Prior knowledge and functionally relevant features in concept learning. Journal of Experimental Psychology: Learning, Memory, \& Cognition, 21, 449-468.

WISNIEWSKI, E. J., \& MEDIN, D. L. (1994). On the interaction of theory and data in concept learning. Cognitive Science, 18, 221-281.

Younger, B. A., \& CoHEN, L. B. (1985). How infants form categories. In G. H. Bower (Ed.), The psychology of learning and motivation (Vol. 19, pp. 211-247). New York: Academic Press.

\section{NOTES}

1. The differences among domains were not of theoretical interest; we report them primarily to ensure that the effects were consistent across the three domains. Across experiments (and in past studies), the effects were usually somewhat weaker for the animal than for the other domains: The subjects seemed somewhat less familiar with some of the differences between predatory and prey animals. Nonetheless, the effects were numerically present for all three domains in every experiment.

2 . The 36 subjects were subdivided into 6 groups ( 3 stimulus domains $\times 2$ knowledge conditions), and so there were only 6 subjects contributing to any single item. Furthermore, because the responses were simply correct or incorrect choices, the data for each item were somewhat variable (i.e., l subject switching a response would make a difference of $17 \%$ for that item) 


\section{APPENDIX}

All of the exemplars in this study consisted of five characteristic features and one idiosyncratic feature. The feature pairs listed below are written in the following form: feature for Category $\mathrm{A} /$ feature for Category $\mathrm{B}$.

Animals, Characteristic Features

Active during the day/Active at night

Has rounded ears/Has pointed ears

Has a long snout/Has a short snout

Striped/Spotted

Can't swim/Can swim

Animals, Idiosyncratic Features (Intact Theme and Mixed Theme)

Can't be domesticated/Can be domesticated

Has sharp teeth/Has flat teeth

Eats meat/Eats plants

Lives alone/Lives in groups

Aggressive/Placid

Has a barbed tail/Has a furry tail

Animals, Idiosyncratic Features (No Theme)

Is endangered/Is not endangered

Has a keen sense of smell/Has a keen sense of hearing

Lives in the Northwest/Lives in the Northeast

Hibernates/Doesn't hibernate

Eats leaves/Eats seeds

Has a four-chambered heart/Has a two-chambered heart

Buildings, Characteristic Features

Has Venetian blinds/Has window shades

Has central heating/Has non-central heating

Has colonial style furniture/Has Victorian style furniture

Has wall-to-wall carpeting/Has rugs

Has a large kitchen/Has a small kitchen

Buildings, Idiosyncratic Features (Intact Theme and Mixed Theme)

Atmospheric research is carried out there/

Deep-sea research is carried out there

Get there by plane/Get there by submarine
Astronauts live there/Divers live there

It floats in the air/It's under the water

Has thin, light walls/Has thick, heavy walls

Birds are kept there as pets/Fish are kept there as pets

Buildings, Idiosyncratic Features (No Theme)

Has a balcony/Has a patio

Has a 6-month lease/Has a 12-month lease

Has a garden in front/Has a fountain in front

Fish are kept there as pets/Birds are kept there as pets

Has a brick exterior/Has a wood exterior

Has a shingled roof/Has a tile roof

Vehicles, Characteristic Features

Has air bags/Has automatic seat belts

Has manual transmission/Has automatic transmission

Has vinyl seat covers/Has cloth seat covers

Has two doors/Has four doors

License plate in front/License plate in back

Vehicles, Idiosyncratic Features (Intact Theme and Mixed Theme)

Used on safaris/Used in mountain climbing

Green/White

Made in Africa/Made in Norway

Lightly insulated/Heavily insulated

Has wheels/Has treads

Drives in jungles/Drives on glaciers

Vehicles, Idiosyncratic Features (No Theme)

Has window stickers/Has bumper stickers

Made in Michigan/Made in Ohio

Has a small trunk/Has a large trunk

Uses gas/Uses diesel

Green/White

Has a cassette player/Has a cd player 